\title{
Unstable neurons underlie a stable learned behavior
}

\author{
William A Liberti III ${ }^{1,2,10}$, Jeffrey E Markowitz ${ }^{1,9,10}$, L Nathan Perkins ${ }^{1,2}$, Derek C Liberti ${ }^{3,4}$, Daniel P Leman ${ }^{1}$, \\ Grigori Guitchounts $^{5,6}$, Tarciso Velho ${ }^{7,8}$, Darrell N Kotton ${ }^{3,4}$, Carlos Lois ${ }^{7}$ \& Timothy J Gardner $^{1}$

\begin{abstract}
Motor skills can be maintained for decades, but the biological basis of this memory persistence remains largely unknown. The zebra finch, for example, sings a highly stereotyped song that is stable for years, but it is not known whether the precise neural patterns underlying song are stable or shift from day to day. Here we demonstrate that the population of projection neurons coding for song in the premotor nucleus, HVC, change from day to day. The most dramatic shifts occur over intervals of sleep. In contrast to the transient participation of excitatory neurons, ensemble measurements dominated by inhibition persist unchanged even after damage to downstream motor nerves. These observations offer a principle of motor stability: spatiotemporal patterns of inhibition can maintain a stable scaffold for motor dynamics while the population of principal neurons that directly drive behavior shift from one day to the next.
\end{abstract}

Questions about coding stability at the single neuron level have been notoriously difficult to address, given the challenge of stably recording from single neurons using implanted electrodes. In the hippocampus, earlier methods tracking tens of cells emphasized stable neural tuning over a timescale of a week ${ }^{1,2}$, whereas recent studies tracking thousands of cells using new optical techniques ${ }^{3,4}$ revealed that $75-85 \%$ of the cells change their tuning properties within the same timeframe ${ }^{5}$. In whisker motor cortex, individual neurons in mice trained on an object detection task were unstable, but the relationship between ensemble measurements and behavior remained stable ${ }^{6}$. These studies support the view that for stable behaviors, individual neurons involved can show substantial drift in their tuning properties.

For motor systems, the stability of neural tuning is not necessarily expected: the convergence of vast numbers of neurons in motor cortex to relatively few muscles ${ }^{7}$ suggests that a given muscular activation pattern could be produced by many patterns of neural activity ${ }^{8}$. In parallel, single neurons in motor cortex are observed to switch tuning properties in a task-dependent manner ${ }^{9}$. In the zebra finch, the precise timing and acoustic structure of song is preserved for years ${ }^{10,11}$. Since a zebra finch sings a single song over the course of his lifetime, the question of neural coding stability is particularly well-defined. In the premotor nucleus that drives song, HVC (used as a proper name), three primary classes of neurons can be found: inhibitory interneurons, basal ganglia-projecting neurons $\left(\mathrm{HVC}_{\mathrm{X}}\right)$ and motor projecting neurons $\left(\mathrm{HVC}_{\mathrm{RA}}\right)$. A third projection neuron type, $\mathrm{HVC}_{\mathrm{AV}}$, sparsely populates the nucleus, but its activity has not yet been described in singing birds ${ }^{12}$. In HVC, each neuron type that has been observed produces a highly stereotyped pattern of action potentials, and these patterns are stable over the time intervals during which they have been recorded: minutes to hours ${ }^{13,14}$. For inhibitory interneurons, the pattern is dense, with spikes occurring throughout song ${ }^{14,15}$. In contrast, excitatory projection neurons that communicate with downstream targets fire "ultra-sparsely"13.

During song production, $\mathrm{HVC}_{\mathrm{X}}$ neurons and inhibitory interneurons exhibit cell-type-specific phase-locking to the local field potential (LFP) at $30 \mathrm{~Hz}$. HVC $\mathrm{X}_{\mathrm{X}}$ cells fire at the peak of the LFP and interneurons at the trough ${ }^{16}$. Intracellular recordings in vitro and in vivo suggest that $\mathrm{HVC}_{\mathrm{RA}}$ neurons also fire during gaps of inhibition ${ }^{17}$. Moreover, both the LFP and projection neuron activity are clustered over a $100-\mu \mathrm{m}$ length-scale. The phase-locking relationship between excitatory and inhibitory neurons is only observed with multiple neuron recordings in the same small volume; phase shifts across HVC preclude a global rhythmic relationship among cell types ${ }^{18}$. Taken together, these observations indicate that spatially coherent mesoscopic patterns of inhibition underlie HVC dynamics, where synchronous gaps in local interneuron population activity control projection neuron timing.

Recent evidence in support of locally organized ensemble activity in HVC allows us to raise the following question: is the persistence of the song motor pattern explained by single neuron stability or by stability of ensemble dynamics? A recent experiment suggests that HVC ensemble dynamics measured through multiunit activity ${ }^{19}$ are resilient to circuit perturbations ${ }^{20}$. Here we extended the observation of HVC ensemble dynamics using new electrophysiological ${ }^{15}$ and imaging methods (Fig. 1) to address the stability of excitatory projection neurons.

\section{RESULTS}

Song is supported by stable mesoscopic dynamics

LFPs can reflect the synchronous activity of neural ensembles over a length-scale of approximately $100 \mu \mathrm{m}^{21}$ (though see ref. 22).

\footnotetext{
${ }^{1}$ Department of Biology, Boston University, Boston, Massachusetts, USA. ${ }^{2}$ Graduate Program in Neuroscience, Boston University, Boston, Massachusetts, USA. ${ }^{3}$ Center for Regenerative Medicine, Boston University School of Medicine, Boston, Massachusetts, USA. ${ }^{4}$ The Pulmonary Center and Department of Medicine, Boston University School of Medicine, Boston, Massachusetts, USA. ${ }^{5}$ Center for Brain Science, Harvard University, Cambridge, Massachusetts, USA. ${ }^{6}$ Program in Neuroscience, Harvard University, Cambridge, Massachusetts, USA. ${ }^{7}$ Division of Biology and Biological Engineering, California Institute of Technology, Pasadena, California, USA. ${ }^{8}$ Brain Institute, Federal University of Rio Grande de Norte, Natal, Brazil. ${ }^{9}$ Present address: Department of Neurobiology, Harvard Medical School, Boston, Massachusetts, USA. 10These authors contributed equally to this work. Correspondence should be addressed to T.J.G. (timothyg@bu.edu).
} 
a
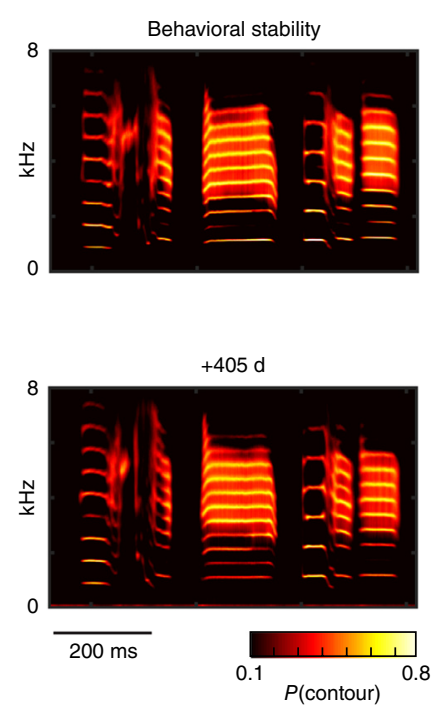

C
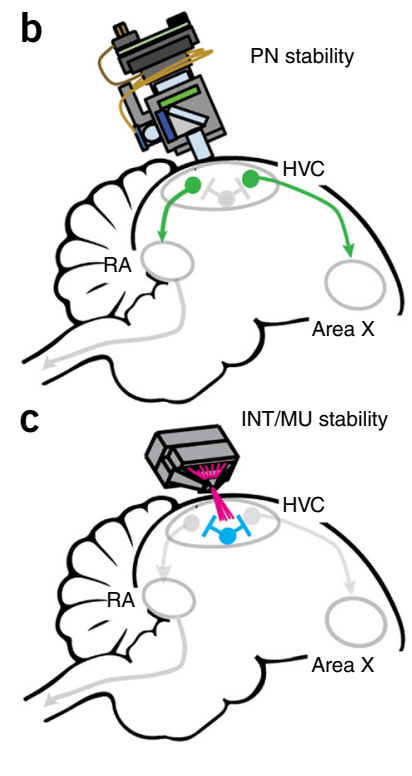

Figure 1 Approach to measuring the stability of neural firing patterns underlying a highly stable behavior. (a) Spectral density images (Online Methods) for the songs sung by the same bird $405 \mathrm{~d}$ apart $(n=1,412$ songs for Day 1 and $n=1,480$ songs for Day 405; audio recordings performed in $n=10$ birds). In this image, the color scale is contour probability, $P$ (contour). (b) To measure projection neuron (PN) stability, a head mounted fluorescence microscope was used to image GCaMP6 delivered by a cell-type-specific virus. (c) Stability of multiunit (MU) and inhibitory interneuron (INT) activity patterns was measured using a custom carbon-fiber electrode array (Online Methods).

We recorded LFPs in the zebra finch premotor nucleus HVC using both commercial and previously described custom carbon-fiber electrode arrays ${ }^{15,16}$ (Online Methods). The phase of the 30-Hz LFP is coherent with the spiking of HVC interneurons and projection neurons (Fig. 2a-f) ${ }^{16}$; we found that this local phase was precisely conserved over long timescales (Fig. 2g). For most of our implants, we were able to record the LFP for up to 30 days, and we found that over this timescale the $30 \mathrm{~Hz}$ phase exhibited a drift of less than 0.25 radians or approximately $15^{\circ}$ (modal change of 0.244 radians over 30 days, from 0.232 to 0.254 ; $95 \%$ bootstrap confidence interval; Supplementary Fig. 1a). This stability in LFP phase indicated that ensemble activity, reflecting a combination of local spiking and presynaptic inputs, did not undergo a major reconfiguration.

Multiunit ensembles and single inhibitory neurons are stable The LFP is thought to reflect ensemble activity from both excitatory and inhibitory neurons over a length-scale of approximately $100 \mu \mathrm{m}$ (ref. 16); similarly, multiunit recordings can reflect the aggregate activity of tens of nearby neurons within a local volume. To monitor the stability of multiunit activity, we implanted bare carbon fibers with a length matched to the full depth of HVC (Online Methods). Figure $\mathbf{2 h}$ shows a multiunit raster with detailed temporal structure in the pattern of firing and silence, with an average precision of $4.587 \mathrm{~ms}$ (4.348-4.843 ms, 95\% bootstrap confidence interval; Online Methods) consistent with the stereotyped neural activity reported previously in $\mathrm{HVC}^{13,23}$. The neural patterns shown in Figure $\mathbf{2 h}$ represent finely timed bursts of activity in an unknown number of cells (the number of simultaneously active cells recorded per electrode was more than could be separated by spike-sorting). However, spike-field measurements indicated that, as for single inhibitory neurons, the multiunit

signal was phase-locked to the trough of the $30 \mathrm{~Hz}$ component of the LFP. This suggests that the multiunit signals were dominated by inhibitory interneurons (Fig. 2c,f $)^{16}$. Enhanced representation of inhibitory cell activity in multiunit signals is to be expected since projection neurons fire "ultra-sparsely"13 and contribute many fewer spikes to a multiunit signal than do interneurons. The suggestion drawn from multiunit signals that patterns of inhibition are stable in HVC (Fig. $\mathbf{2 h}$-j) was directly confirmed in long-term single unit recordings from $n=6$ inhibitory neurons from single unit recordings (3 are shown in Fig. 2k). As revealed in Figure 2k, inhibitory neuron firing patterns were remarkably stable, even over a time-scale of months. In addition to the stability of the $30-\mathrm{Hz}$ LFP phase, this result indicated that ensemble inhibitory activity was highly stable.

We next asked if the stability of this multiunit activity depended on sensory feedback. Previous work has shown that the statistical distribution of postsynaptic potentials in HVC projection neurons is invariant to altered auditory feedback induced through a tracheosyringeal (TS) nerve cut ${ }^{24}$, but over long time-scales, the song of the adult zebra finch is known to be maintained through an auditoryfeedback-dependent process ${ }^{25,26}$. We anticipated that the rate of drift in the ensemble pattern would increase with perturbations to auditory feedback. Following baseline recording, we severed the ipsilateral TS nerve, which relays motor commands to the birds' vocal organ $(n=5$ birds; Online Methods). As in previous work ${ }^{24,27}$, this loss of muscular control results in acoustic distortions of song (Fig. 3a-d and see Supplementary Fig. 2 for all examples), degrading the learned sensory-motor correspondence between vocal commands and auditory feedback. Following this, we monitored the song and HVC multiunit firing patterns for a period of approximately 1 month. Over this time interval, song did not recover, and the multiunit spike patterns in the premotor nucleus HVC remained stable (Fig. 3e-f). In all birds subject to the TS cut, shifts in the firing pattern occurred at the same rate as for baseline recordings $(P>0.05$ for all birds, one-tailed Wilcoxon rank-sum test; Online Methods). We conclude from this that the ensemble pattern in HVC was robust to changes in auditory feedback that result from unilateral TS nerve removal. Here the song was altered, but the detailed ensemble firing patterns persisted in HVC as though no change had occurred. This observation is synergistic with a recent study showing resilience of the HVC ensemble pattern after neural circuit perturbations ${ }^{20}$.

\section{Individual projection neurons are unstable over days}

Our electrophysiology methods were typically unable to track individual excitatory projection cells over time-scales longer than a single day, perhaps due to the limitations of recording from smaller cells ${ }^{15}$. To provide information about the stability of excitatory cells, we turned to optical imaging of genetically encoded calcium indicators (Fig. 4a, Supplementary Fig. 3 and Online Methods) ${ }^{16,28}$ using a virus that labels excitatory projection neurons in HVC but does not label inhibitory neurons (Online Methods). Imaging during singing was accomplished through the use of miniature head-mounted microscopes ${ }^{3}$ using both a commercial head-mounted microscope (Inscopix, $n=1$ bird) and a custom 3D-printed microscope ( $n=3$ birds) designed to promote freedom of movement in singing birds (Fig. 4, Supplementary Fig. 4 and Online Methods). The custom microscope provided, to our knowledge, the first measurements of calcium activity during undirected singing, the solo practice song of the zebra finch. Projection neuron calcium activity patterns were sparse and time-locked to singing (Fig. 4a). The timing of most projection neurons was stable over several days (Fig. 5a,b); however, across days, the probability that a given cell fired during singing could 

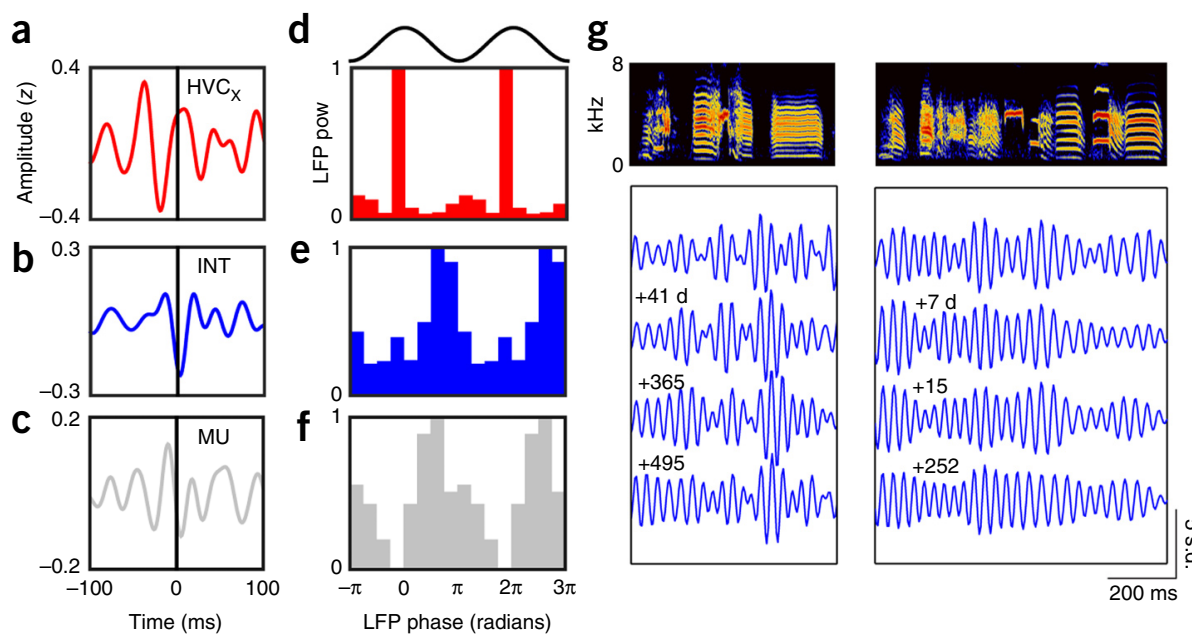

h
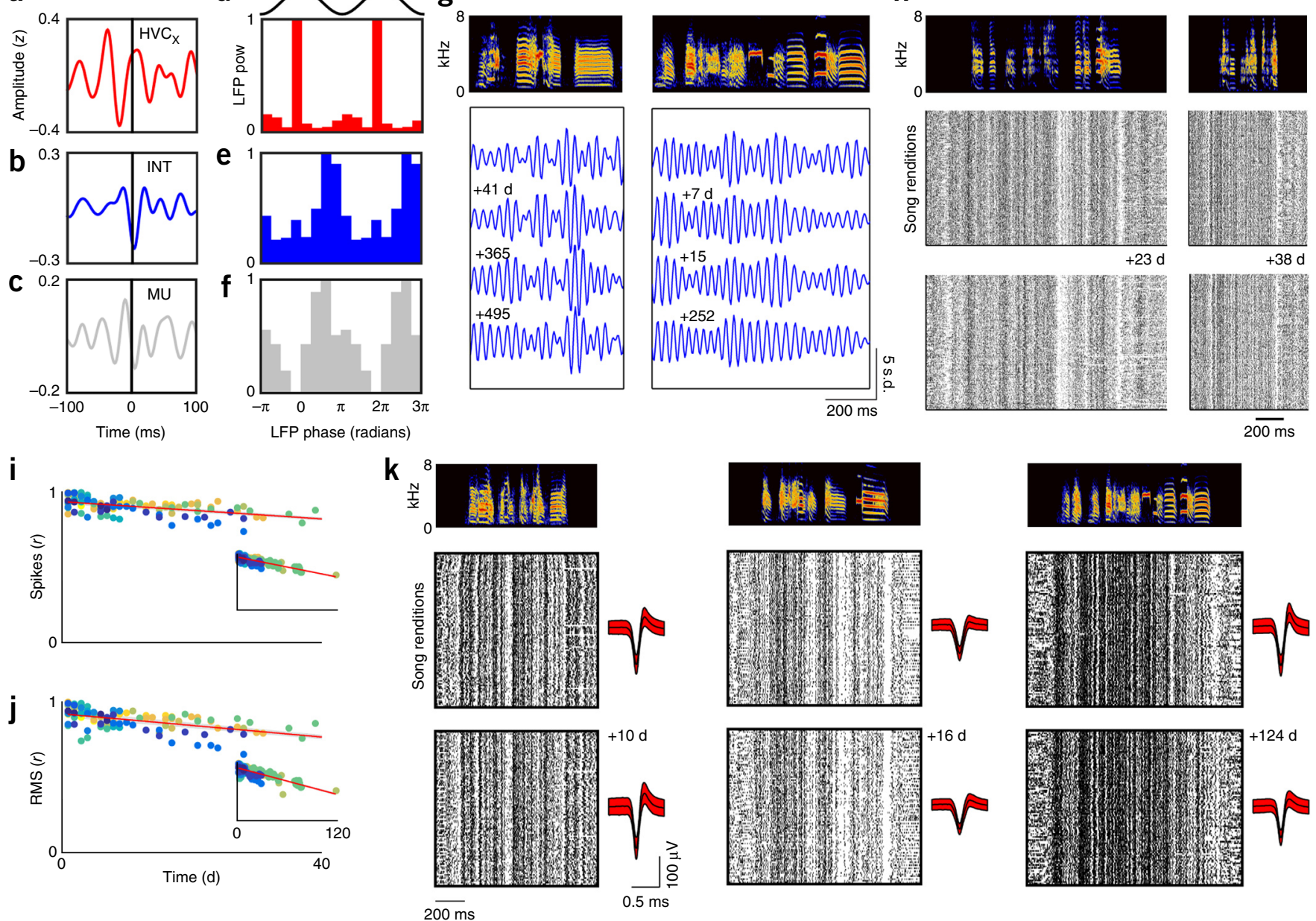

Figure 2 Activity patterns from multiunit ensembles and single interneurons are highly stable. (a-c) Burst-triggered averages of 25-35 Hz LFPs for (a) $\mathrm{HVC}_{\mathrm{X}}$ neurons (average of $n=12$ neurons) (b) interneurons (average of $n=65$ neurons) and (c) multiunit activity (average of $n=3$ sites with both multiunit and LFP recordings). The $y$-axis is in units of $z$-score. (d-f) Histograms showing the power of the LFP binned at the phase of the LFP for each burst time. A clear separation in preferred phase can be seen between (d) HVC $C_{X}$ neurons ( $n=12$ neurons) and (e) interneurons ( $n=65$ neurons) but not (f) between interneurons and multiunit activity ( $n=3$ sites with both multiunit and LFP recordings). (g) Top, example song spectrogram; bottom, examples of trial-averaged band-passed LFPs $(25-35 \mathrm{~Hz})$ from a single electrode in two different birds. Here the LFP was trial-averaged and $z$-scored on each day of the recording (LFP recordings repeated across $n=182$ channels from 21 implants). The left example was recorded up to $495 \mathrm{~d}$ after the implant. Vertical scale bar, s.d., is standard deviation. (h) Examples of multiunit stability over month-long time-scales (multiunits recorded from $n=20$ sites). Top, example song spectrogram; bottom, spike raster of song-aligned trials. (i,j) Multiunit patterns are stable over $40 \mathrm{~d}$, measured using patterns in (i) firing rate (regression slope $=-0.0028$ ) or (j) root-mean-square (RMS; regression slope $=-0.0038$ ) (insets: $120 \mathrm{~d}$ ). Shown are average correlations between each day and the first day; red line indicates linear regression and shading indicates $99 \%$ confidence interval of fitted values ( $n=130$ values from 20 birds). Colors indicate different birds, many not visible because they overlap in the upper left portion of the plot. (k) Three example inhibitory interneuron firing patterns (of $n=6$ recordings lasting more than $24 \mathrm{~h}$ ). The right-most example is stable for up to $124 \mathrm{~d}$. Top, example song spectrogram; bottom, spike raster of song-aligned trials. Average waveforms from each day are shown next to the corresponding raster; red shading indicates s.d.

change dramatically (Fig. 5c and Supplementary Fig. 5). Across an interval of days, cells both dropped out and newly appeared in the song pattern (Fig. 5b,c and Supplementary Figs. 6-8). Stable and unstable regions of interest (ROIs) could be found next to each other (Fig. 5c and Supplementary Figs. 6 and 7).

One class of projection neuron $\left(\mathrm{HVC}_{\mathrm{X}}\right)$ can produce more than one time-locked burst of action potentials during singing. For cells with multiple timing peaks, amplitude or probability of activation could change independently for each peak. This was observed both in calcium imaging and in electrophysiological recordings, where we witnessed the 'fade-in' of a second burst time in a projection neuron recorded with a carbon-fiber electrode over the course of a day (Fig. 5d).

For the imaging data, we performed analysis on two ROI sets: one analysis included all ROIs found on any day of imaging

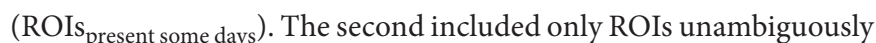
present on all days of imaging (ROIs present all days ). By the fifth day of imaging, across all ROIs recorded, a large number of ROIs showed statistically significant changes in their mean song-aligned activity $(P<0.01$ permutation test, ROIs present some days: 15 of 39 ROIs for bird 1, 27 of 38

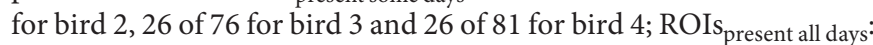
11 of 18,14 of 16,11 of 22 and 5 of 21 , respectively).

The shift in projection neuron activity across multiple days contrasted with our electrophysiological recordings, which typically revealed stability of projection firing patterns over the course of hours within a single day ${ }^{13,15,29}$ (Fig. 6a,b). This contrast motivated us to examine whether the shift in projection neuron activity occurred over intervals of sleep (Fig. 6c-f). We found a much stronger shift in songaligned projection neuron calcium traces after a night of sleep than 
a
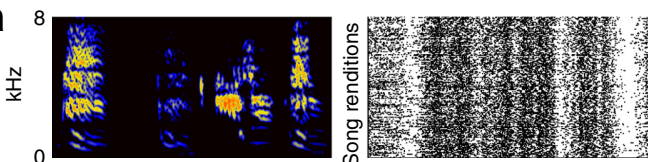

b
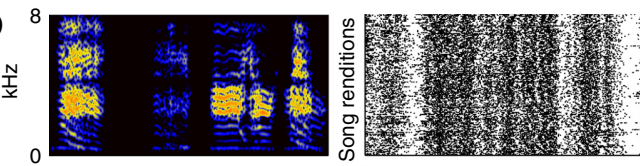

$200 \mathrm{~ms}$

C Acoustic change after cut, $\mathbf{e}$
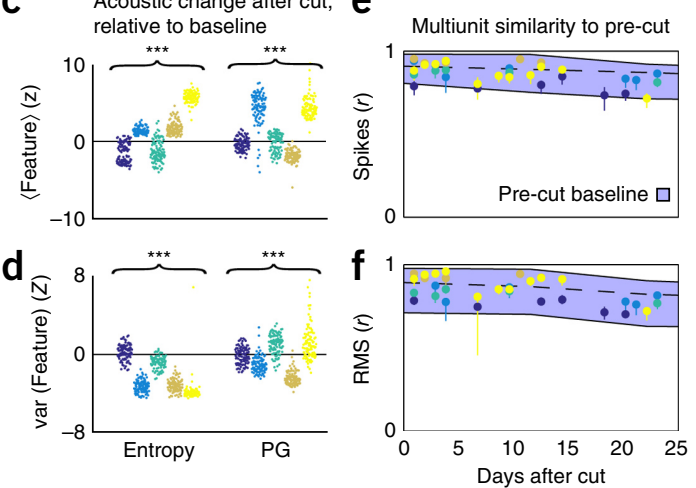

Figure 3 Drift in multiunit firing patterns is not accelerated by unilateral TS nerve transection. (a,b) Representative song spectrograms, left, and multiunit rasters, right, (a) before and (b) after the TS nerve cut (experiment performed in $n=5$ birds). (c,d) Change in acoustic features after nerve cut $(* * * P<0.001$, Wilcoxon rank-sum test, $\langle$ Entropy $\rangle$ $P=1.9 \times 10^{-15}, z=-7.95 ;\langle\mathrm{PG}\rangle P=5.7 \times 10^{-5}, z=-4.02 ; \operatorname{var}($ Entropy) $P=0, z=16.25 ; \operatorname{var}(P G) P=2.6 \times 10^{-5}, z=4.21 ; n=100$ songs each from 5 birds; Online Methods). PG, pitch goodness. (e,f) After the nerve cut, multiunit activity did not drift more than expected in the precut baseline condition ( $n=34$ time points from 5 birds; see Online Methods for exact $P$-values). Shown is the average correlation at each timepoint relative to the last baseline day. Blue shading, the $99 \%$ bootstrap confidence interval estimated from the baseline condition.

over the course of a single day (Fig. 6c and Supplementary Fig. 6; ROIs present some days, $P=2.0 \times 10^{-40}, z=-13.26$; ROIs present all days, $P=1.6 \times 10^{-14}, z=-7.59$, one-tailed Wilcoxon signed-rank test). Next, we checked if drift could be accounted for by time elapsed. First, we analyzed whether calcium activity decreased in consistency across the day and, unexpectedly, found the opposite to be true: consistency

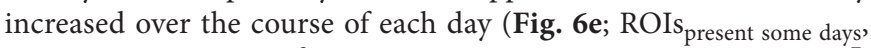
$r=0.14, P=6.1 \times 10^{-9}$; ROIs $s_{\text {present all days }}, r=0.18, P=9.7 \times 10^{-7}$; Online Methods). We also found that song-aligned calcium activity became more similar to the previous day's average over the course of the day (Fig. 6f; ROIs present some days $, r=0.12, P=5.6 \times 10^{-3}$; ROIs $s_{\text {present all days, }} r=0.25, P=4.3 \times 10^{-9}$, Spearman rank-correlation test). Thus, calcium traces in single cells changed both their mean pattern and variance overnight, and they returned toward the previous day's average as the day progressed, while at the same time becoming more stereotyped. Given these observations, the drift in calcium activity was not simply a function of time elapsed. Rather, these results suggest that sleep actively destabilized the representation of song in premotor nucleus HVC.

\section{The microstructure of song changes overnight and increases in consistency throughout the day}

The finding that projection neuron activity was unstable across days prompted us to examine the microstructure of song behavior using a highly sensitive time-frequency analysis method developed for analysis a

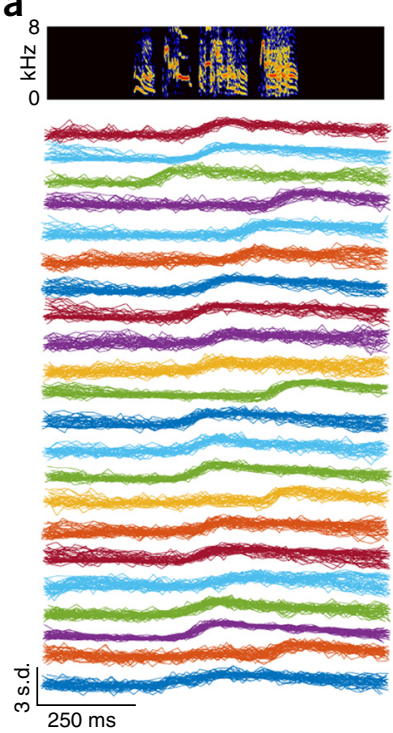

b

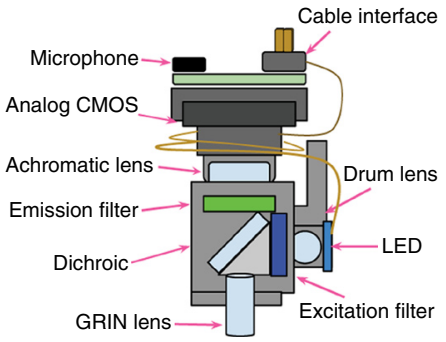

C

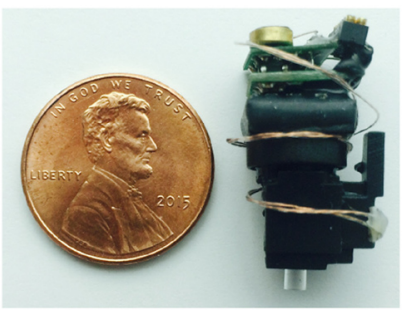

Figure 4 A custom head-mounted fluorescence microscope adapted for use in singing birds. (a) Top, spectrogram of a single song rendition; bottom, calcium traces from 22 ROls over 50 song-aligned trials in a single bird. Vertical scale bar, s.d., indicates standard deviation. Imaging performed in $n=4$ birds. (b) Schematic of the miniature microscope used for this study (Online Methods). (c) Photograph of the 3D printed microscope.

of sparse signals such as zebra finch song (Online Methods). The persistence of zebra finch song structure has been documented over time-scales of years ${ }^{10,11}$ and as demonstrated in Figure 1, over the period of a year, the song appears to be remarkably stable (Fig. 1a). However, the microstructure of song is not precisely the same, even in this example. High-resolution investigation of the microstructure revealed small but significant shifts over the interval of a week, and these changes occurred primarily over periods of sleep $\left(P=9.8 \mathrm{e} \times 10^{-4}\right.$, $w=55$, one-tailed Wilcoxon signed-rank test similarity scores for $n=10$ birds; Supplementary Figure 9 and Online Methods). Moreover, similarly to the drift in calcium activity, we found that song consistency also increased over the course of the day (Fig. $\mathbf{6 d} ; r=0.28$, $P=6.6 \times 10^{-8}$, Spearman rank-correlation test; Online Methods).

\section{DISCUSSION}

This study suggests that motor skills encoded in the brain, like many structural features of the human body, undergo renewal at the cellular level. Skin maintains its shape despite the turnover of cells ${ }^{30}$, and the intestinal surface has constant turnover but maintains its function ${ }^{31}$. For the stable song of the zebra finch, the instability of the neural program qualitatively matched the microscopic day-to-day changes in song, but the song changes observed over intervals of sleep were small, while the pattern of neuronal participation changed more dramatically. What explains the persistence of the song motor pattern in spite of the unstable projection neurons underlying song?

A key finding is that the premotor nucleus HVC is stable on a mesoscopic scale. Over the length scales recorded in LFPs and multiunit recordings (approximately $100 \mu \mathrm{m}$ ), the neural basis of song is largely unchanged over a time-scale of weeks to years. In contrast, individual projection neurons drift, not primarily in the timing of their activity as much as in the burst probability and participation in the song pattern. One potential factor contributing to ensemble stability in HVC is the spatial correlation observed in the firing patterns of excitatory cells. Nearby excitatory neurons in HVC fire at similar 

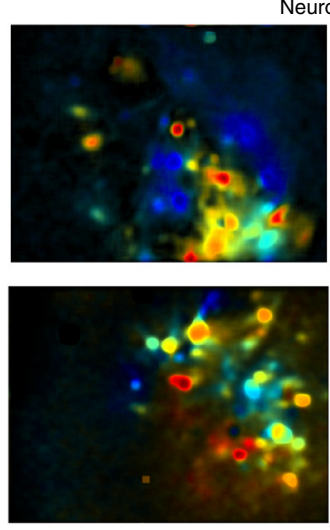

b

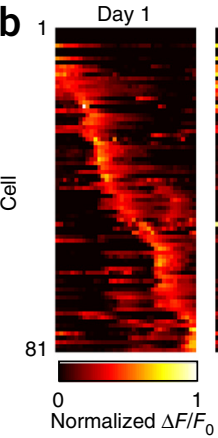

Neuron timing stability
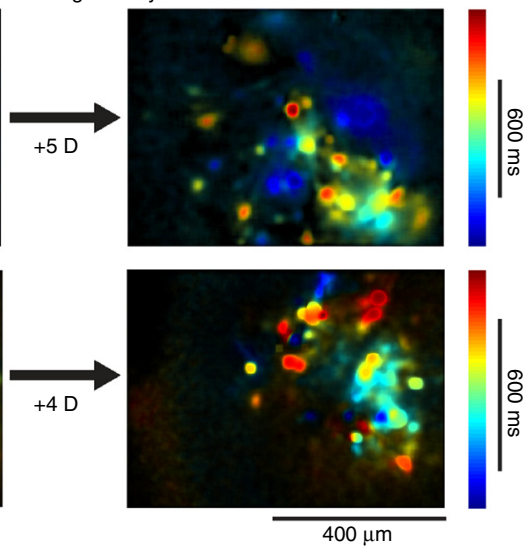

C
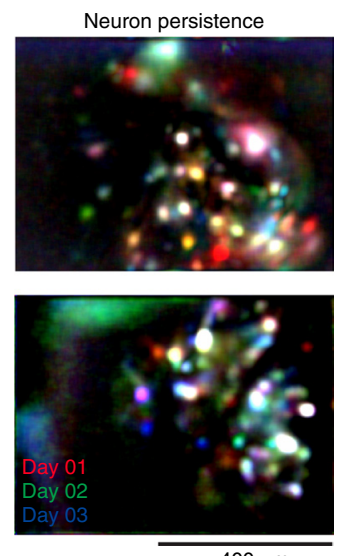

$400 \mu \mathrm{m}$
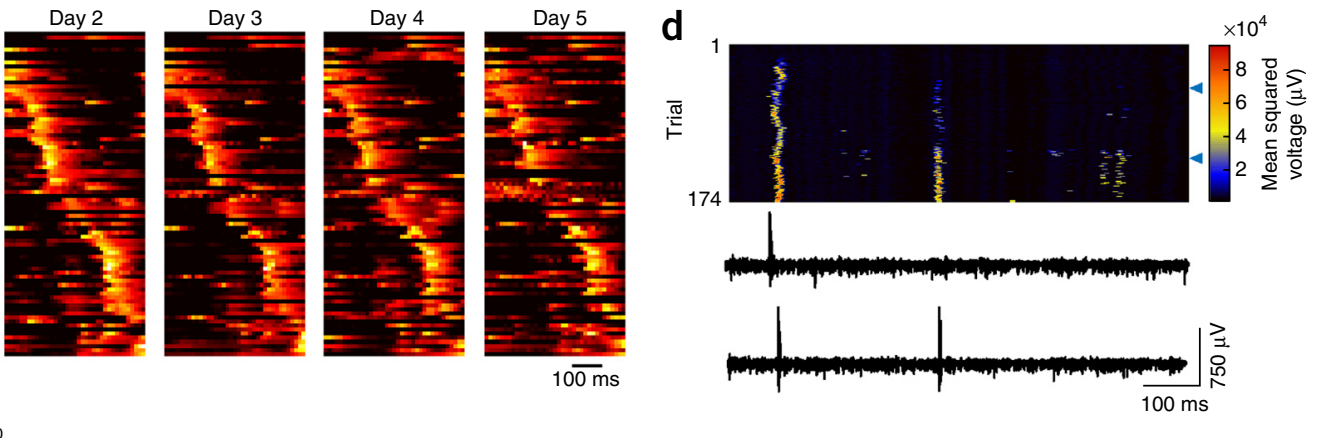

Figure 5 Projection neurons drift over a 5-d imaging session. (a) Images colored by the timing of max pixel intensity, for 2 birds, across 5 -d (top, commercial microscope) and 4-d (bottom, custom microscope) intervals (imaging performed in $n=4$ birds). (b) Trial-averaged activity from all songrelated neurons from 1 animal. Keeping the same sorting and amplitude color scale, the ROls are plotted for 5 consecutive d. (c) For the same animals shown in a, cells active on 3 consecutive $d$ are combined into a single image in which color indicates neuron participation rather than timing. (red = Day 1 , blue $=$ Day 2, green = Day 3). Cells with persistent activity on all 3 d appear white in this representation (Supplementary Fig. 5). Cells that changed amplitude or changed firing probability appear colored. (d) Electrophysiological recording of a projection neuron reveals a new song-locked burst, which emerged over the course of a day. Blue triangles indicate trials plotted at the bottom of the figure.

times, and the length-scale of this correlation is roughly $100 \mu \mathrm{m}$ (ref. 16). If individual projection neurons fade out of the ensemble, nearby projection neurons could be serving redundant roles.

A second related factor contributing to ensemble stability is the role of temporally patterned inhibition in HVC. In contrast to the instability of excitatory neuron coding, we have observed that inhibitory interneuron firing patterns are stable for weeks or months: for as long as it was technically possible to track the firing patterns of the cells. At the simplest level, excitatory neurons are postulated to be driven by a relatively sparse number of strong synapses, leading to the sparsity of their firing patterns ${ }^{29,32}$. In contrast, many inhibitory interneurons in HVC have large dendritic arbors and are thought to be driven by a large number of presynaptic partners ${ }^{33}$. The prediction from HVC connectivity is that changes in a small number of synapses could alter the excitatory neuron firing patterns dramatically but influence the inhibitory interneurons very little. In light of this numerical argument and the observed stability of single inhibitory neurons, HVC ensemble patterns could be stabilized if interneurons have a strong local influence over projection neuron activity. Indeed, this appears to be the case. The firing times of the excitatory projection neurons coincide with stereotyped pauses in local inhibition ${ }^{17,34}$, and $\mathrm{HVC}_{\mathrm{X}}$ excitatory neurons and inhibitory interneurons fire in opposite phases of the $30-\mathrm{Hz} \mathrm{LFP}{ }^{16}$. The observations are true on the microscale, but due to phase shifts across the extent of HVC the rhythmic alternation is not observed globally ${ }^{18}$. Local blockade of inhibition releases the sparse-firing cells from inhibition, and they begin to fire at multiple times in song ${ }^{17}$. Building on these observations, a recent modeling study suggested that inhibitory interneuron dynamics can increase the robustness of HVC dynamics to added noise ${ }^{35}$. These models can be made more precise in future experiments as additional cell-type-specific information is measured. The relative stability of different classes of $\mathrm{HVC}$ excitatory neurons $\left(\mathrm{HVC}_{\mathrm{RA}}, \mathrm{HVC}_{\mathrm{X}}\right.$ and $\mathrm{HVC}_{\mathrm{AV}}$ ) remain to be examined, since our methods could not distinguish among the three cell types. Stable, patterned inhibition in the songbird may be an important force in maintaining the dynamical pattern of song in spite of underlying drift in the projection neurons. For mammals, similar principles may apply. Aspects of the song corticothalamic loop resemble features of mammalian motor cortex, including an important $30 \mathrm{~Hz}$ rhythm ${ }^{16,36,37}$ and spatial correlations in neuron firing patterns over $100-\mu \mathrm{m}$ length scales ${ }^{38-40}$. In mammalian motor cortex, it may be fruitful to track drift in excitatory versus inhibitory neuron populations to see if patterned inhibition may provide a stabilizing force in motor skill persistence.

Finally, we addressed the importance of sleep in the rearrangement of the song code in HVC. The change in the song motor pattern occurred primarily over intervals of sleep, and we showed that adult song behavior also underwent microscopic shifts in sleep. Our results may be related to a previous observation: as juveniles learn to sing, their songs progress through a day of practice ${ }^{41}$ but degrade slightly over intervals of sleep ${ }^{42}$. The depth of this overnight 'backsliding' 

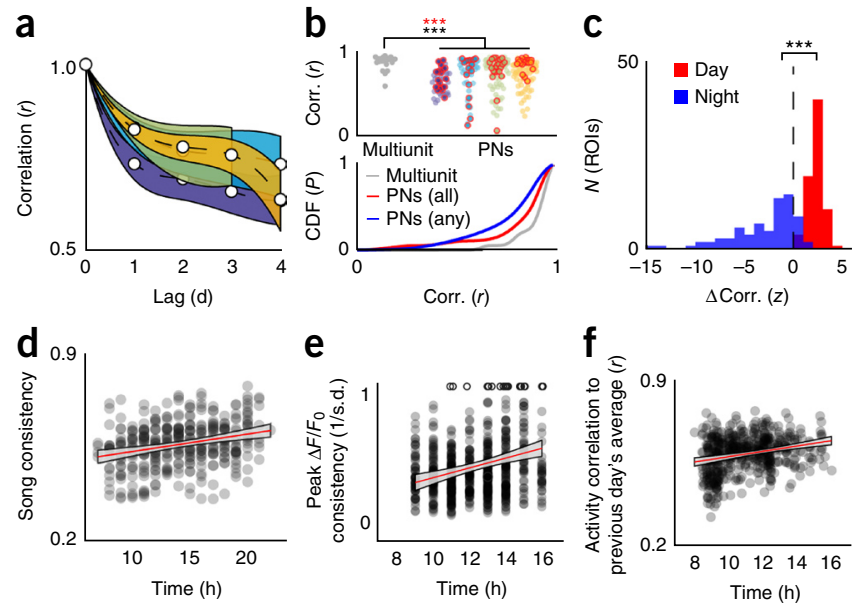

Figure 6 Projection neuron activity preferentially changed over periods of sleep. (a) Average correlation across all ROIs across different lags for each bird; each color indicates a different bird. Shaded region indicates $99 \%$ bootstrap confidence interval (ROIs present some days). (b) Top, all correlations (Corr.) up to a lag of $4 \mathrm{~d}$ for multiunit activity ( $n=20$ birds, 1 sample per bird) and projection neuron activity (ROIs sresent some days, $P=2.7 \times 10^{-6}, z=4.70$, one-tailed Wilcoxon rank-sum test, $n=234$ ROIs from 4 birds). Red, cells that participated unambiguously across the entire time series (ROls present all days, $P=9.8 \times 10^{-4}, z=3.30$, one-tailed Wilcoxon rank-sum test, $n=77$ ROIs from 4 birds). Bottom, cumulative density functions (CDF) of the data shown above: multiunit data in gray, projection neurons seen across all days in red and projection neurons seen clearly on any day in blue. (c) The shift in correlation ( $\Delta$ Corr.) occurs primarily overnight (ROIs sresent some days, $P=2.0 \times 10^{-40}, z=-13.26$; ROls present all days, $P=1.6 \times 10^{-14}, z=-7.59$, Wilcoxon signed-rank test; shown are data from ROIs present all days). (d) Song consistency increased over the course of the day $\left(r=0.28, P=6.6 \times 10^{-8}\right.$, Spearman rank correlation, $n=372$ values from 10 birds; Online Methods). (e) Consistency in the amplitude of peak $\Delta F / F_{0}$ (measured over the whole song) increased with time of day (ROIspresent some days, $r=0.14, P=6.1 \times 10^{-9}, n=1,769$ values; ROls present all days, $r=0.18, P=9.7 \times 10^{-7}, n=708$ values, Spearman rank correlation; Online Methods; shown are data from ROIs present all days). Unfilled circles indicate values $>1$. (f) The trial-by-trial correlation between calcium activity and the previous day's average also increased with time of day (ROIs present some days, $r=0.12, P=5.6 \times 10^{-3}, n=529$ values; ROIs present all days, $r=0.25, P=4.3 \times 10^{-9}, n=540$ values, Spearman rank correlation; shown are data from ROls present all days). All time-of-day measurements in $\mathbf{e}$ and $\mathbf{f}$ use only data from our custom microscopes, detailed in Figure 4 (data from 3 birds; $n=153$ ROIspresent some days; $n=56$

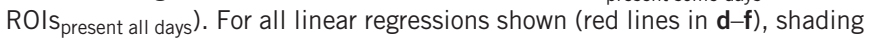
indicates $99 \%$ confidence interval of the fitted values. ${ }^{* *} P<0.001$.

is positively correlated with the eventual success of song learning, indicating that nighttime song rearrangements are important for learning. In addition, if song-aligned HVC activity is disrupted through lesioning Nif, an area upstream of $\mathrm{HVC}$, the pattern recovers primarily overnight ${ }^{20}$.

The present study provides a possible neural mechanism for these sleep effects, raising the possibility that new patterns of activity 'invented' over intervals of sleep provide important raw material for song learning and maintenance. Francis Crick proposed that noisy reactivation of neural circuits in sleep weakens the strongest pathways in the brain, promoting adaptive plasticity ${ }^{43}$. It is thought that in sleep, songbirds reactivate their song patterns in the motor region RA in a noisy or incomplete manner ${ }^{44}$, and it is possible that this spontaneous activity drives the overnight shift in HVC excitatory neurons observed here. Future studies may track the firing patterns of excitatory neurons in $\mathrm{HVC}$ while silencing or overstimulating spontaneous activity in sleep, directly testing the hypothesis that noisy replay of song in sleep promotes adaptive plasticity of the song motor program. It also remains to be seen whether the overnight shift in HVC is random or guided by vocal errors. Random shifts in the population could increase robustness of HVC dynamics by enforcing redundant representations of song. In contrast, shifts in the population of neurons that are influenced by vocal performance errors could be an active part of song learning and maintenance.

\section{METHODS}

Methods, including statements of data availability and any associated accession codes and references, are available in the online version of the paper.

Note: Any Supplementary Information and Source Data files are available in the online version of the paper.

\section{ACKNOWLEDGMENTS}

The authors thank H. Eichenbaum and the Center for Neuroscience at BU for the loan of the Inscopix microscope. Special thanks to D.S. Kim and L. Looger for providing the GCaMP6 DNA and to the GENIE project at Janelia Farm Research Campus, Howard Hughes Medical Institute. This work was supported by a grant from CELEST, an NSF Science of Learning Center (SBE-0354378) and by grants from NINDS (R01-NS089679-01 and 1U01NS090454-01).

\section{AUTHOR CONTRIBUTIONS}

W.A.L., J.E.M., G.G. and D.P.L. performed the experiment; J.E.M. and W.A.L. analyzed the data; L.N.P. provided software for the custom microscope; D.C.L., D.N.K., T.V. and C.L. provided the lentivirus; T.V. and C.L. discovered the cell-type-specificity of the viral vector. W.A.L., J.E.M. and T.J.G. designed the experiment and wrote the manuscript.

\section{COMPETING FINANCIAL INTERESTS}

The authors declare no competing financial interests.

Reprints and permissions information is available online at http://www.nature.com/ reprints/index.html.

1. Thompson, L.T. \& Best, P.J. Long-term stability of the place-field activity of single units recorded from the dorsal hippocampus of freely behaving rats. Brain Res. 509, 299-308 (1990).

2. Kentros, C.G., Agnihotri, N.T., Streater, S., Hawkins, R.D. \& Kandel, E.R. Increased attention to spatial context increases both place field stability and spatial memory. Neuron 42, 283-295 (2004).

3. Ghosh, K.K. et al. Miniaturized integration of a fluorescence microscope. Nat. Methods 8, 871-878 (2011).

4. Chen, T.-W. et al. Ultrasensitive fluorescent proteins for imaging neuronal activity. Nature 499, 295-300 (2013).

5. Ziv, Y. et al. Long-term dynamics of CA1 hippocampal place codes. Nat. Neurosci. 16, 264-266 (2013).

6. Huber, D. et al. Multiple dynamic representations in the motor cortex during sensorimotor learning. Nature 484, 473-478 (2012).

7. Leonardo, A. Degenerate coding in neural systems. J. Comp. Physiol. A Neuroethol. Sens. Neural Behav. Physiol. 191, 995-1010 (2005).

8. Rokni, U., Richardson, A.G., Bizzi, E. \& Seung, H.S. Motor learning with unstable neural representations. Neuron 54, 653-666 (2007).

9. Ganguly, K. \& Carmena, J.M. Emergence of a stable cortical map for neuroprosthetic control. PLoS Biol. 7, e1000153 (2009)

10. Immelmann, K. Song development in the zebra finch and other estrildid finches. in Bird Vocalizations (ed. Hinde, R.A.) 61-74 (Cambridge University Press, 1969).

11. Lombardino, A.J. \& Nottebohm, F. Age at deafening affects the stability of learned song in adult male zebra finches. J. Neurosci. 20, 5054-5064 (2000).

12. Akutagawa, E. \& Konishi, M. New brain pathways found in the vocal control system of a songbird. J. Comp. Neurol. 518, 3086-3100 (2010).

13. Hahnloser, R.H.R., Kozhevnikov, A.A. \& Fee, M.S. An ultra-sparse code underlies the generation of neural sequences in a songbird. Nature 419, 65-70 (2002).

14. Kozhevnikov, A.A. \& Fee, M.S. Singing-related activity of identified HVC neurons in the zebra finch. J. Neurophysiol. 97, 4271-4283 (2007).

15. Guitchounts, G., Markowitz, J.E., Liberti, W.A. \& Gardner, T.J. A carbon-fiber electrode array for long-term neural recording. J. Neural Eng. 10, 046016 (2013).

16. Markowitz, J.E. et al. Mesoscopic patterns of neural activity support songbird cortical sequences. PLOS Biol. 13, e1002158 (2015).

17. Kosche, G., Vallentin, D. \& Long, M.A. Interplay of inhibition and excitation shapes a premotor neural sequence. J. Neurosci. 35, 1217-1227 (2015). 
18. Lynch, G.F., Okubo, T.S., Hanuschkin, A., Hahnloser, R.H.R. \& Fee, M.S. Rhythmic continuous-time coding in the songbird analog of vocal motor cortex. Neuron 90 , 877-892 (2016).

19. Crandall, S.R., Aoki, N. \& Nick, T.A. Developmental modulation of the temporal relationship between brain and behavior. J. Neurophysiol. 97, 806-816 (2007).

20. Otchy, T.M. et al. Acute off-target effects of neural circuit manipulations. Nature 528, 358-363 (2015)

21. Katzner, S. et al. Local origin of field potentials in visual cortex. Neuron 61, 35-41 (2009).

22. Buzsáki, G. Anastassiou, C.A. \& Koch, C. The origin of extracellular fields and currents--EEG, ECoG, LFP and spikes. Nat. Rev. Neurosci. 13, 407-420 (2012).

23. Schmidt, M.F. Pattern of interhemispheric synchronization in HVc during singing correlates with key transitions in the song pattern. J. Neurophysiol. 90, 3931-3949 (2003).

24. Vallentin, D. \& Long, M.A. Motor origin of precise synaptic inputs onto forebrain neurons driving a skilled behavior. J. Neurosci. 35, 299-307 (2015).

25. Nordeen, K.W. \& Nordeen, E.J. Auditory feedback is necessary for the maintenance of stereotyped song in adult zebra finches. Behav. Neural Biol. 57, 58-66 (1992).

26. Tschida, K.A. \& Mooney, R. Deafening drives cell-type-specific changes to dendritic spines in a sensorimotor nucleus important to learned vocalizations. Neuron 73, 1028-1039 (2012).

27. Williams, H., Crane, L.A., Hale, T.K., Esposito, M.A. \& Nottebohm, F. Right-side dominance for song control in the zebra finch. J. Neurobiol. 23, 1006-1020 (1992).

28. Picardo, M.A. et al. Population-level representation of a temporal sequence underlying song production in the zebra finch. Neuron 90, 866-876 (2016).

29. Long, M.A., Jin, D.Z. \& Fee, M.S. Support for a synaptic chain model of neuronal sequence generation. Nature 468, 394-399 (2010).

30. Weinstein, G.D. \& Van Scott, E.J. Autoradiographic analysis of turnover times of normal and psoriatic epidermis. J. Invest. Dermatol. 45, 257-262 (1965).

31. Creamer, B., Shorter, R.G. \& Bamforth, J. The turnover and shedding of epithelial cells. The turnover in the gastro-intestinal tract. Gut 2, 110-118 (1961).
32. Diesmann, M., Gewaltig, M.O. \& Aertsen, A. Stable propagation of synchronous spiking in cortical neural networks. Nature 402, 529-533 (1999).

33. Mooney, R. \& Prather, J.F. The HVC microcircuit: the synaptic basis for interaction between song motor and vocal plasticity pathways. J. Neurosci. 25, 1952-1964 (2005).

34. Amador, A., Perl, Y.S., Mindlin, G.B. \& Margoliash, D. Elemental gesture dynamics are encoded by song premotor cortical neurons. Nature 495, 59-64 (2013).

35. Cannon, J., Kopell, N., Gardner, T. \& Markowitz, J. Neural sequence generation using spatiotemporal patterns of inhibition. PLoS Comput. Biol. 11, e1004581 (2015).

36. Rubino, D., Robbins, K.A. \& Hatsopoulos, N.G. Propagating waves mediate information transfer in the motor cortex. Nat. Neurosci. 9, 1549-1557 (2006).

37. Murthy, V.N. \& Fetz, E.E. Coherent 25- to $35-\mathrm{Hz}$ oscillations in the sensorimotor cortex of awake behaving monkeys. Proc. Natl. Acad. Sci. USA 89, 5670-5674 (1992).

38. Dombeck, D.A., Graziano, M.S. \& Tank, D.W. Functional clustering of neurons in motor cortex determined by cellular resolution imaging in awake behaving mice. J. Neurosci. 29, 13751-13760 (2009).

39. Peters, A.J., Chen, S.X. \& Komiyama, T. Emergence of reproducible spatiotemporal activity during motor learning. Nature 510, 263-267 (2014).

40. Hira, R. et al. Spatiotemporal dynamics of functional clusters of neurons in the mouse motor cortex during a voluntary movement. J. Neurosci. 33, 1377-1390 (2013).

41. Ohgushi, E., Mori, C. \& Wada, K. Diurnal oscillation of vocal development associated with clustered singing by juvenile songbirds. J. Exp. Biol. 218, 2260-2268 (2015)

42. Derégnaucourt, S., Mitra, P.P., Fehér, O., Pytte, C. \& Tchernichovski, O. How sleep affects the developmental learning of bird song. Nature 433, 710-716 (2005).

43. Crick, F. \& Mitchison, G. The function of dream sleep. Nature 304, 111-114 (1983).

44. Dave, A.S. \& Margoliash, D. Song replay during sleep and computational rules for sensorimotor vocal learning. Science 290, 812-816 (2000). 


\section{ONLINE METHODS}

Subjects. All procedures were approved by the Institutional Animal Care and Use Committee of Boston University (protocol numbers 14-028 and 14-029). Electrophysiology data were collected from $n=27$ adult male zebra finches $(>120 \mathrm{DPH})$, and imaging data were collected from $n=4$ adult male zebra finches. Birds were individually housed for the entire duration of the experiment and kept on a 14-h light-dark cycle. The birds were not used in any other experiments.

Splaying microfiber electrodes. We used a previously described minimally invasive carbon fiber electrode array ${ }^{15}$ in addition to commercially available arrays (TDT, Neuronexus) to chronically monitor both single units and LFPs. Extracellular voltages were amplified and digitized at either 25 or $30 \mathrm{KHz}$ using the Intan acquisition system (RHA2000 and RHD2000).

Microfiber electrodes for multiunit recordings. Bare (i.e., uncoated) microthread electrodes were prepared by extracting 5 - and $11-\mu \mathrm{m}$ diameter fibers from commercially available spools of carbon fiber (grade XAS, HTA, T300 or P25). Once extracted, sizing and other impurities were removed by heating fibers as previously described ${ }^{15}$. The bare fibers were then attached to coated silver wire using a conductive silver paint (842-20G, MG Chemicals). Signals were sent from a custom headstage to a differential amplifier (AM Systems 1700, gain of 1,000; low cut-off, $300 \mathrm{~Hz}$; high cut-off, $5 \mathrm{kHz}$ ) and digitized with a National Instruments Acquisition board (PCIE 6323, 40-kHz sampling rate).

Tracheosyringeal nerve cut. The right TS nerve was removed using previously described methods ${ }^{27}$. Briefly, birds were anesthetized with $4 \%$ isoflurane and maintained at $1-2 \%$ for the course of the surgery. Feathers were removed from the neck, and an incision was made over the trachea. The nerve was dissected from the surrounding tissue and the nerve was pulled from its roots on the syrinx, extracting a minimum of $1 \mathrm{~cm}$ of nerve length.

Calcium imaging. To image calcium activity in HVC projection neurons during singing, we employed head-mounted fluorescence microscopes, both commercial and custom built. This method provides long-term recordings of calcium signals in $\mathrm{HVC}^{16}$ and enables studies of motor stability and adaptive plasticity at the single neuron level. For viral delivery, we used the genetically encoded calcium indicators GCaMP6s ( 2 birds) and GCaMP6f ( 2 birds) delivered by lentiviruses ${ }^{16,45}$.

For 1 bird, we used a commercial microscope (Inscopix) to gather femaledirected singing over 5-d periods. However, these microscopes could not be used with a rotary commutator and were too heavy to evoke reliable undirected or solo song. Motivated by these challenges, we developed a lightweight $(\sim 1.7 \mathrm{~g})$, commutable, 3D-printed single-photon fluorescent microscope that simultaneously records audio and video (Fig. 4 and Supplementary Fig. $4 ; n=3$ birds). These microscopes enabled recording of hundreds of songs per day (all birds sang at least 500 songs on at least one day of recording), and all songs were recorded from birds longitudinally in their home cage, without requiring adjustment or removal of the microscope during the imaging period. Birds were imaged for less than $20 \mathrm{~min}$ total on each imaging day, and LED activation and video acquisition were triggered on song using previous described methods. All analysis was restricted to a particular bout of song, either the first ( 1 bird) or the second ( 3 birds). Recordings were taken from 3 weeks to 3 months after virus injection.

Microscope design. The custom microscope is similar to a previously described design $^{3}$ (Fig. 4 and Supplementary Fig. 4). A blue LED produces excitation light (470-nm peak, LUXEON Rebel). A drum lens collects the LED emission, which passes through a $4 \mathrm{~mm} \times 4 \mathrm{~mm}$ excitation filter, deflects off a dichroic mirror and enters the imaging pathway via a 0.25 pitch gradient refractive index (GRIN) objective lens. Fluorescence from the sample returns through the objective, the dichroic, an emission filter and an achromatic doublet lens that focuses the image onto an analog CMOS sensor with $640 \times 480$ pixels mounted on a PCB that also integrates a microphone. The frame rate of the camera is $30 \mathrm{~Hz}$, and the field of view is approximately $800 \mu \mathrm{m} \times 600 \mu \mathrm{m}$. The housing is made of 3D-printed material (Formlabs, black resin). A total of 5 electrical wires run out from the camera: one wire each for camera power, ground, audio, NTSC analog video and LED power. These wires run through a custom flex-PCB interconnect
(Rigiflex) up to a custom-built active commutator, based on previously described designs ${ }^{46}$. The NTSC video signal and analog audio are digitized through a USB frame-grabber. Custom software written in the Swift programming language running on the Mac OS X operating system (version 10.10) leverages native AVFoundation frameworks to communicate with the USB frame-grabber and capture the synchronized audio-video stream. Video and audio are written to disk in MPEG-4 container files with video encoded at full resolution using either H.264 or lossless MJPEG Open DML codecs and audio encoded using the AAC codec with a $48-\mathrm{kHz}$ sampling rate. In addition, the software communicates with a microcontroller via a USB-to-serial connection to manipulate LED intensity and samples a trigger signal from a DSP performing song detection (TDT RX8) in order to selectively record during singing.

Viruses. Addgene plasmids \# 40753 (pGP-CMV-GCaMP6s) and \# 40755 (pGPCMV-GCaMP6f), a gift from the Douglas Kim laboratory, were transformed into E. coli bacteria by heat shock and were sequenced. The GCaMP6s and GCaMP6f fragments were PCR amplified with the addition of NotI/BamH1 to the $5^{\prime}$ and $3^{\prime}$ ends respectively. GCaMP6s was cloned into the pHAGE-CMV-eGFP vector to form pHAGE-CMV-GCaMP6s. The RSV promoter sequence was ordered from IDT as a gblock with the addition of SpeI/NotI to the $5^{\prime}$ and $3^{\prime}$ ends respectively and was cloned into pHAGE-CMV-GCaMP6s and pHAGE-CMV-eGFP to form pHAGE-RSV-GCaMP6s and pHAGE-RSV-eGFP. GCaMP6f was then cloned into pHAGE-RSV-GCaMP6s to form pHAGE-RSV-GCaMP6f. The viruses were packaged in HEK293T cells and titered on FG293 cells, with titers ranging from $1.2-2.3 \times 10^{10}$ infectious particles/ml. Plasmid maps and sequences of all lentiviral vectors employed can be downloaded from the vectors page of the laboratory of Darrel Kotton (http://www.kottonlab.com). These plasmids have also been deposited for distribution on Addgene (http://www.addgene.com/Darrell_Kotton) as plasmid IDs \#80315 and \#80146.

The tropism of our virus for excitatory neurons was evaluated by counterstaining using known markers of inhibitory neurons, specifically anti-parvalbumin (Abcam, Ab11427), anti-calretinin (SWANT, 7697) and anti-calbindin (SWANT, CB-300). Out of 1,000 counted neurons, only 1 was double-labeled with inhibitory markers. Confocal imaging of GFP-positive cells revealed that in all cases where dendrites were visible they contained spines, consistent with the known morphology of $\mathrm{HVC}_{\mathrm{RA}}$ and $\mathrm{HVC}_{\mathrm{X}}$ projection neurons. The morphology of all GFPpositive cells was consistent with previous descriptions of projection neurons in HVC but not consistent with either glial cells or inhibitory neurons. Our histology also revealed dense axon arbors in nucleus RA and Area $\mathrm{X}$, indicating that a significant number of $\mathrm{HVC}_{\mathrm{RA}}$ and $\mathrm{HVC}_{\mathrm{X}}$ neurons were labeled (Supplementary Fig. 3). The combined weight of these observations suggests that lenti-RSV overwhelmingly labels projection neurons in HVC. However, it remains unclear if the excitatory neuron tropism results from the RSV promoter, if it is a general tropism of the lentivirus construct itself, or if it is a combination of both.

Data Analysis. Song alignments. Songs were aligned using the Euclidean distance in spectral features between the data and a template song in a sliding window. Local minima in the Euclidean distance were considered candidate hits, which were then plotted in 2 dimensions for the user to perform a cluster cut. No time warping was applied to any data. These methods have been described in more detail previously ${ }^{47}$.

Spectral density images. To plot the variability of multiple song renditions in time and frequency we used the spectral density image, which has been described previously ${ }^{16,48}$. A sparse binary time-frequency representation was generated using auditory contours ${ }^{49,50}$. These time-frequency binary images were combined by averaging across all renditions. In the resulting image, the value at each pixel is the probability that a time-frequency contour passes through it.

Acoustic changes after the nerve cut. To analyze the change in song after the TS nerve cut, we computed amplitude modulation (AM), frequency modulation (FM), Wiener entropy, amplitude envelope, pitch and pitch goodness using Sound Analysis Pro for Matlab ${ }^{51}$. We then computed the average or variance of each feature across the entire song and compared the last day of singing precut with the first day of singing after the cut.

Acoustic change overnight. To quantify changes in song microstructure, we used the similarity score derived from the spectral density image, which has been described previously ${ }^{48}$. For 5 consecutive $\mathrm{d}$ of singing from $n=10$ birds, 
we divided each day's worth of singing in half by trial number. To compute the change in song microstructure across the day, we computed the similarity scores between trials from the first half of the day and the spectral density image from the second half of the day. Then, to analyze the overnight change, we computed similarity scores between trials from the first half of each day and the spectral density image from the previous evening. Finally the scores were averaged for each day before statistical comparison. Next, to estimate the change in song consistency over the course of the day (Fig. 6d), we computed the spectral density image for each hour's worth of singing. Then, similarity scores were calculated for each trial and the corresponding spectral density image. Values were averaged in $1-\mathrm{h}$ time bins. Trends were estimated by taking the Spearman rank correlation between similarity score and the time of day.

Local field potentials and single units. Local field potentials (LFPs) were analyzed as described previously ${ }^{16}$. Extracellular voltage traces were median filtered (1-ms window) to remove spikes and then low-pass filtered with a $400-\mathrm{Hz}$ corner frequency (4th order Butterworth filter) and downsampled to $1 \mathrm{kHz}$. Finally, the LFP was filtered with a $25-35-\mathrm{Hz}$ bandpass (8th order Elliptic filter for Figure 2; 53-tap Kaiser window FIR filter, 20-dB stop-band attenuation, 0.05 ripple for all analysis to minimize the impulse response). To compute the change in phase angle, we used the angle of the Hilbert-transformed narrowband LFP.

Single interneurons were analyzed as described previously ${ }^{16}$. Extracellular voltage traces were bandpass-filtered from $11-600 \mathrm{kHz}$ (12th order Elliptic filter, $0.2-\mathrm{dB}$ pass-band ripple, $40-\mathrm{dB}$ stop-band attenuation) and sorted using standard offline spike sorting techniques ${ }^{52,53}$.

Multiunit electrophysiology. First, threshold crossings were taken from extracellular traces digitized at $40 \mathrm{kHz}$ using a threshold of 2.5 robust s.d. ${ }^{54}$ Threshold crossings were then converted into firing rates on a single-trial basis by convolving with a Gaussian kernel (5-ms sigma). Alternatively, the rootmean-square (RMS) of the voltage signal was calculated in a 5-ms sliding window (box car). To estimate the stability of multiunit activity, we averaged either the song-aligned firing rate or the RMS across all trials in a given day. Then, the Pearson correlation coefficient was computed between the averages from either the first day of recording or the last day pre-TS cut and each subsequent day (Fig. 2i-j). To compare the drift after the TS cut to the baseline condition, correlation values were binned in a 16 - $\mathrm{d}$ sliding window (5-d overlap; the result was insensitive to binning parameters; data not shown), and a bootstrap test was performed between the binned pre- and post-nerve cut correlation values (Fig. 3e-f). That is, binned post-nerve cut values were compared with 1,000 bootstrap estimates of the corresponding pre-nerve cut correlation values. Exact $P$-values were (from smallest to largest, both spikes and RMS): $P=0.075,0.11$, $0.22,0.42,0.58$ and 0.74 ; all others $P=1$.

Calcium imaging. Time plots used in Figure 5a were created by averaging all song-aligned calcium imaging movies for a bird within a single day. The resulting 'average' movie was smoothed with a $15-\mu \mathrm{m}$ Gaussian filter, and each pixel was then colored by its center of mass in time. To create the presence or absence images used in Figure 5c, maximum projection images were created for all song-aligned calcium imaging movies, and the average pixel intensity of these maximum projections was taken for each day of imaging. Each day was mapped to red, green or blue. For Supplementary Figure 7c, each maximum projection image was divided by a smoothed version of the same image, using a $100-\mu \mathrm{m}$ pixel disk filter, to normalize across bright and dim ROIs.

Region of interest (ROI)-based analysis was performed as described previously ${ }^{16}$. In brief, raw imaging data was motion-corrected using a previously published algorithm ${ }^{55}$. ROIs were manually selected and pixels were averaged for each frame within each ROI. ROI traces were converted to $\Delta F / F_{0}$ by estimating $\mathrm{F}_{0}$ as the 12th percentile in an 800 -ms sliding window. Drift was assessed by computing the Pearson correlation between trial-averaged calcium traces for each ROI at all possible lags. Correlation values from the same lag (for example, between Days 1 and 2 and between Days 2 and 3 ) were averaged for each ROI (a similar procedure was used for the multiunit data comparison in Figure $\mathbf{6 b}$ ). To account for any uncertainty in the alignments due to the $30-\mathrm{Hz}$ sampling rate of the camera, we then computed the maximum Pearson correlation in a $100-\mathrm{ms}$ window. Drift was then quantified using a randomization permutation test with a $P=0.01$ threshold. Specifically, we compared the correlations observed at each lag (1-4 d) with correlations from the same data with the group (i.e. day) labels scrambled. $P$-values were determined by estimating the probability that the observed correlation was greater than or equal to the correlation values from over 10,000 randomizations. We also repeated this analysis using the timing of peak $\Delta F / F_{0}$ on each day (not shown). Specifically, for each ROI the time of peak trial-averaged $\Delta F / F_{0}$ was computed on each day, and the peak times were compared between each day of imaging and the first. For each cell, if the peak timing changed by more than $100 \mathrm{~ms}$ or there were no peaks greater than $0.5 \% \Delta F / F_{0}$, the cell was considered unstable.

In order to compare within-day to overnight changes in correlations, we split the data on each day in halves by trial number. The within-day correlations were measured by computing the correlations between trial-averages using the first and second half of trials from a given day. The overnight correlations were measured by computing the correlations between trial-averages from the second half of one day with the first half of the subsequent day. The within-day and overnight correlations were averaged across days for each ROI, and subsequently a Wilcoxon signed-rank test was used to compare the two groups of correlation values. For visualization (Fig. 6c) the two populations were $z$-scored using a bootstrap.

To test for significant interactions between time of day and the consistency of calcium activity, we first computed the amplitude of peak $\Delta F / F_{0}$ across the entire song for each ROI (this controlled for any change in song duration across the day). Next, we computed the s.d. of the peak $\Delta F / F_{0}$ in 1-h bins and computed the Spearman rank correlation between these values and time of day. To account for changes in peak $\Delta F / F_{0}$ resulting from photobleaching, we computed the partial rank correlation between the time of day and s.d. of the peak $\Delta F / F_{0}$ after accounting for the correlation between time of day and mean of the peak $\Delta F / F_{0}$ (again using 1-h bins). Then, to analyze the similarity of calcium activity to the previous day's average, we peak-normalized the $\Delta F / F_{0}$ values for each trial through dividing by the median peak $\Delta F / F_{0}$ determined using the 5 nearest trials (with respect to time of day). Then, we estimated the similarity between each trial and the previous day's average using the peak Pearson correlation coefficient (also over a 100-ms window). Finally, we computed the Spearman rank correlation between these similarity values and time of day.

Controls for stability of the imaging plane and photobleaching. Across days, ROIs were manually inspected and adjusted to ensure that the center of mass for each ROI was at the center of the ROI mask and that the mask did not overlap with neighboring cells. Masks that were moved more than the diameter of the mask at any point of the longitudinal study were excluded from analysis. To ensure that our results were not impacted by the stability of the imaging plane, we then checked to see if there were any differences in the spatial profile of stable and unstable ROIs. More precisely, we computed the $\Delta F / F_{0}$-weighted centroid within each ROI for each day. Then, we took the Euclidean distance between the $\Delta F / F_{0}$-weighted centroids for each ROI across consecutive days of imaging. Lastly, the distances for unstable and stable ROIs (determined using the permutation test described above) were compared on each day of imaging (for example, for the same ROI imaged on $4 \mathrm{~d}$ there would be 3 distances measured from day to day) and we found no significant effect (ROIs present some days, $P=0.17, z=0.97$, $n=164$ and $n=620$ comparisons made for stable and unstable, respectively; ROIs $_{\text {present all days }}, P=0.99 z=-4.86, n=84$ and $n=180$ comparisons for stable and unstable, respectively; one-tailed Wilcoxon rank-sum test). This indicated that micromotion of cells in the imaging plane did not correlate with their shift in firing patterns. Moreover, to control for any effects of bleaching in our time of day analysis we repeated the analysis of calcium activity consistency using the Spearman rank partial correlation accounting for correlation between time of day and average peak $\Delta F / F_{0}$. A significant effect was still observed (ROIs present some days, $r=0.12, P=7.8 \times 10^{-7}, n=1,769$; ROIs $s_{\text {present all days }}$, $\left.r=0.17, P=2.8 \times 10^{-6}, n=708\right)$.

Statistical analysis. No formal methods were used to predetermine sample sizes; the sample sizes used here are similar to those used in the field. No randomization of experimental sessions was performed, and no blinding to experiment condition was performed during the analysis. All statistical comparisons were performed using nonparametric tests (Wilcoxon rank-sum, Wilcoxon signed-rank, bootstrap or randomization tests). Where appropriate, we controlled for multiple comparisons using the Holm-Bonferroni step-down procedure.

A Supplementary Methods Checklist is available.

Data Availability. The data that support the findings of this study are available from the corresponding author upon reasonable request. The latest version of 
the custom Matlab scripts used for analysis in this manuscript are available at https://github.com/gardner-lab/HVC-Stability-Analysis.

45. Wilson, A.A. et al. Sustained expression of alpha1-antitrypsin after transplantation of manipulated hematopoietic stem cells. Am. J. Respir. Cell Mol. Biol. 39, 133-141 (2008)

46. Fee, M.S. \& Leonardo, A. Miniature motorized microdrive and commutator system for chronic neural recording in small animals. J. Neurosci. Methods 112, 83-94 (2001).

47. Poole, B., Markowitz, J.E. \& Gardner, T.J. The song must go on: resilience of the songbird vocal motor pathway. PLoS One 7, e38173 (2012).

48. Markowitz, J.E., Ivie, E., Kligler, L. \& Gardner, T.J. Long-range order in canary song. PLoS Comput. Biol 9, e1003052 (2013).

49. Lim, Y., Shinn-Cunningham, B. \& Gardner, T.J. Stable Time-Frequency Contours for Sparse Signal Representation 1-5 (EUSIPCO, 2013).
50. Aoi, M., Lepage, K., Lim, Y., Eden, U.T. \& Gardner, T.J. An approach to time-frequency analysis with ridges of the continuous chirplet transform. IEEE Transactions on Signal Processing 63, 699-710 (2015).

51. Tchernichovski, O., Nottebohm, F., Ho, C.E., Pesaran, B. \& Mitra, P.P. A procedure for an automated measurement of song similarity. Anim. Behav. 59, 1167-1176 (2000).

52. Sahani, M. Latent Variable Models for Neural Data Analysis. PhD Thesis, (California Institute of Technology, Pasadena, California, USA, 1999).

53. Tolias, A.S. et al. Recording chronically from the same neurons in awake, behaving primates. J. Neurophysiol 98, 3780-3790 (2007).

54. Quiroga, R.Q., Nadasdy, Z. \& Ben-Shaul, Y. Unsupervised spike detection and sorting with wavelets and superparamagnetic clustering. Neural Comput. 16, 1661-1687 (2004).

55. Guizar-Sicairos, M., Thurman, S.T. \& Fienup, J.R. Efficient subpixel image registration algorithms. Opt. Lett. 33, 156-158 (2008). 